University and Max Planck Institute posted a letter rejecting claims of remote transfer from training in a cognitive task (http://longevity3.stanford.edu/blog/2014/10/15/). This letter brought a reply from Michael Merzenich and a large number of other researchers (http:// www.cognitivetrainingdata.org/) citing empirical evidence for transfer in human and animal studies, although little is yet known about how this improvement in performance takes place.

Most people agree that training improves the task being trained. Reaction time is a particularly important measure of performance in many skills and it is generally thought that over a wide range of conditions there is a monotonic relationship between number of practice trials and reaction time to perform the task (Anderson, Ficham \& Douglass, 1999; Fitts \& Posner, 1967; Heathcote, Brown \& Mewhort, 2000; Newell \& Rosenbloom, 1981). The shape of the function has been disputed, with some arguing for a power function (Anderson et al 1999; Fitts \& Posner, 1967; Newell \& Rosenbloom, 1981) and others an exponential function (Heathcote et al., 2000), but both agree there is a monotonic improvement in reaction time with training.

A deeper understanding of how training improves performance on the trained task may help us gain perspective on the issues of how far such training may assist general cognition. Thus this paper is designed to put a somewhat broader perspective on transfer by considering what is known or might be learned about the cellular and molecular mechanisms influencing improvement in reaction time with training. We hope this approach might also illuminate the issue of the relationship between training effects and transfer to new tasks.

\section{Forms of training}

We have suggested that network and state training are two different methods for training the brain (Tang \& Posner, 2014). These two methods fit with two types of MRI studies. The older imaging approach involves scanning the brain during performance of a task (Posner \& Raichle, 1994). Generally two conditions are compared, one while performing the complete task and a second in which the person performs some part of the task (control condition). Subtraction of the control condition from the complete task condition is designed to isolate operations involved in the first condition that are not present in the second condition. A different MRI method is to examine brain areas whose activation is correlated when the person is not performing any task (Raichle, 2009). These reflect the ongoing state of the brain (default state) without instruction to perform a task.

The first method of training involves using a specific cognitive task (Tang \& Posner, 2014). Two of the most studied are training in working memory (Buschkuehl, Hernandez-Garcia, Jaeggi, Bernard, \& Jonides; 2014) and executive attention (Rueda, Checa \& Combita, 2012), but specific networks related to visual perception (Zatorre, Fields, Johansen-Berg, 2012), reading (Schlaggar \& McCandliss, 2007) and arithmetic (Ansari, 2008) are also well studied. The second method involves training to produce a brain state that might lead to improved performance on many specific networks. The most explored forms of state training have been physical training (Hillman, Erickson, \& Kramer, 2008) and meditation (Tang \& Posner, 2014). 


\section{Brain Changes}

Most of us have learned to think about white matter changes as occurring in child development but not in adults. However, in recent years this view has been altered by findings that dormant oligodendroctyes can be activated when people suffer from a demyelinating disease (Beirowski, 2013). In addition, many forms of training in adults have been shown to result in changes in the efficiency of white matter connections as measured by Diffusion Tensor Imaging with MRI (Wang \& Young, 2014; Zatorre et al., 2012).

There is also evidence from a study of mice learning to run on a wheel with irregular spokes, requiring acquisition of a new and complex motor skill (McKenzie et al., 2014). This learning has also been shown to activate dormant oligodendrocytes. (McKenzie, et al., 2014). When activation of the dormant oligodendrocytes was blocked, the animals did not learn, showing the importance of cells producing myelin for acquiring a motor skill.

The mechanism for white matter change seems to be a general one requiring repeated activation of the pathways associated with training (Gibson et al., 2014). Similar effects of learning on white matter have been reported in human subjects (for a review see Wang \& Young, 2014) using a variety of network and state training methods. These include meditation (Tang \& Posner, 2014), juggling (Scholz, Klein, Behrens, \& Johansen-Berg, 2009) and working memory training (Takeuch et al., 2010). Most of these tasks show change in a statistic called Fractional Anisotropy (FA) measured by Diffusion Tensor Imaging (DTI). This statistic measures the directionality of water molecules and is thought to reflect the efficiency of the white matter tracts along which the molecules move. In some studies changes are measured in axial diffusivity (AD) which is thought to relate mainly to the density of axons within the pathway. Measures of radial diffusivity (RD) have been related in animal studies to changes in myelination (Fields, 2008, 2015; Zatorre et al., 2012).

Working memory training has usually involved later frontal and parietal areas usually including the dorsolateral prefrontal cortex and superior and inferior parietal lobe.

Meditation training has generally involved differences in medial frontal areas including the ventral anterior cingulate. In our studies we have used relaxation training as a control. The more dorsal or cognitive control areas of the anterior cingulate are activated more by the relaxation training than by meditation. This probably reflects the strong conscious control as the person tries to relax each muscle group.

Meditation training has been shown to change white matter (Tang et al., 2012). With respect to the practice of meditation, a current puzzle is how a purely mental activity like control of attention through mindfulness training could influence white matter axonal and myelination changes. We previously developed a working hypothesis (Posner, Tang \& Lynch, 2014) that views frontal theta rhythm described below as releasing a protease to influence the activity of dormant oligodendrocytes or precursor cells.

To test these ideas we are using transgenic mice expressing light-sensitive receptors that allow for temporally precise activation of neurons in the anterior cingulate cortex, a method known as optogenetics (Fenno, Yizar \& Deisseroth, 2011). We targeted this structure based 
on our human data showing white matter changes in a number of pathways surrounding the anterior cingulate following one month of meditation training (Tang et al., 2010).

The standard EEG taken from scalp electrodes can be analyzed to examine the power in various frequency bands. Best known to most people is the alpha rhythm which is around 10 $\mathrm{Hz}$ and is often associated with quiet rest and relaxation. The theta rhythm usually around 4$8 \mathrm{~Hz}$ has been can be recorded strongly from the hippocampus. It has been associated with an alert and oriented state and with the form of synaptic plasticity called long term potentiation (Larson, Wong \& Lynch, 1986). Long term potentiation is probably the most discussed form of plasticity in neuroscience of learning.

There is considerable evidence that meditation training produces a frontal theta rhythm (Xue, Tang, Tang, \& Posner, 2014). Frontal theta is also produced during tasks involving cognitive control (Jiang, Zhang \& van Gaal, 2015; Wolmelsdorf, Johnson, Vinck, \& Everling, 2010) and is associated with plastic changes in learning and memory. Based on these findings we are currently examining the effects of inducing theta and other rhythms on mouse behavior and myelination. We are using optogenetics to stimulate excitatory activity phase-locked to 1,8 or $40 \mathrm{~Hz}$ rhythms for half an hour each day for 30 days. We expect to find changes due to theta and perhaps other rhythms. If we find these changes they may relate to prior findings that long term potentiation (LTP), a major brain mechanism related to learning, can be induced in hippocampal cells following theta stimulation (Larson, Wong, \& Lynch, 1986). In accord with recent evidence for activity dependent myelination (Fields, 2015), repeated stimulation of a broad population of cells via theta might influence overall connectivity.

Although we hypothesized a unique place for theta rhythm, recent studies suggest that even direct current (DC) stimulation can induce or improve a frontal theta rhythm (Miller, Berger \& Sauseng, 2015; Reinhart, Zhu, Park, \& Woodman, 2015). There have also been findings that DC stimulation over frontal electrodes can improve learning (Cohen Kadosh, Soskic, Iuculano, Kanai, Walsh, 2010; Rhinehart et al. 2015). However, there is still as much dispute over the efficacy of DC stimulation as there is for the general proposition that some forms of network specific learning can transfer to a wide range of subsequent tasks (Horvath, Forte, Carter, 2015). We believe that the lack of specific mechanistic explanations, either for producing the far transfer sometimes found in network training or for producing the facilitatory effect of frontal DC stimulation on learning, has made it difficult to establish their links to plasticity. We hope the direction of work described in this paper might help to provide the needed mechanisms.

\section{Individual Differences}

While almost all persons improve on a trained task, the rate of training can vary among persons. How does this occur? Most of the quantitative accounts of how reaction time improves are strictly at the psychological level. They propose priming of specific subroutines (Anderson et al., 1999) or the elimination of inefficient strategies of performing the task (Fitts \& Posner, 1967). However, It has been argued that the development of myelination is regulated by environmental influences on expression of DNA through 
epigenetics (Emory \& Liu, 2015). Although various strategies, backgrounds, motivation etc. are clearly involved in the improvement of RT with training, our paper in this special issue (Voelker, Sheese, Rothbart \& Posner, 2015) presents evidence that differences in the efficiency of methylation may also be important in the rate of improvement. We report that an allelic variation in a gene related to improved methylation can influence the rate of learning in 7 year old children when practicing the Attention Network Test. We found that those children with the higher efficiency allele of the methylenetetrahydrofolate reductase gene (MTHFR) showed greater improvement in reaction time over three sessions of practice on the Attention Network Test (ANT).

The Voelker et al (2016) paper is limited to 7 year old children, but we have subsequently found that adults with the allele linked to higher efficiency methylation show significantly faster RTs for resolution of conflict in the ANT than those with the lower efficiency alleles of MTHFR (Voelker, Rothbart \& Posner, 2016). This finding extends to implicit and explicit performance in a quite different serial reaction time task (Curran \& Keele, 1993), but MTHFR alleles are not related to learning of a paired associate learning task in which reaction time is not an important measure. We believe that the improved performance in both development and in learning results from the effects of methylation on neural networks through changing dopamine release or increasing the efficiency of white matter tracts. The effect of development between age 7 and adulthood produces 10 times greater effect on RT (400 millisec) than the effect of learning at each age (40 millisec). We believe this reflects the lack of myelination in childhood as well as the lengthy period of development between ages 7 and adulthood compared to the brief learning period we studied in children and adults.

The effect of variation in MTHFR was associated with variation in genes regulating neurotransmitter activity. For example, individual differences in executive function involved an interaction between the MTHFR gene and catechol $O$-methyltransferase (COMT) gene. The COMT gene product metabolizes synaptic dopamine and functional variation in COMT has been associated with differences in brain activity during an executive task (Osinsky, Hewig, Alexander \& Hennig, 2012). We also identified individual differences in reaction time across sessions attributed to differences in alerting as a gene $\times$ gene effect of MTHFR and a variation in dopamine $\beta$-hydroxylase (DBH), whose product converts dopamine to norepinephrine. The change in alertness underlies the tendency of children to show an upswing in reaction time late in practice (Voelker et al., 2015). In this study we measure alertness by changes in reaction time due to a warning signal using the Attention Network Test (ANT). In the ANT a warning signal occurs at the center of the screen and indicates a target will be presented in .5 second, but since the target never occurs at fixation and no indication is given about the location of the target (either above or below fixation) no orienting cue is provided.

Differences in neurotransmitter activity in task-relevant pathways may differentially induce the process of myelination via activity-dependent methylation. Our observations that individual differences in learning are related to both neurotransmitter activity and methylation efficiency support this view. 
Our effort to study genetic and epigenetic influence in humans relies on an approach using functional polymorphisms within genes such as MTHFR. Unfortunately, it is impossible with this method to be sure that the influence of MTHFR on the efficiency of white matter causes the RT differences we have found, since MTHFR influences other processes throughout the brain and in other places in the body. In addition, we do not know which cell types and brain areas are critical to our observations. To move further we have begun using our mouse model to better understand these mechanisms at the molecular level.

\section{Implication for Transfer}

Many cognitive tasks and skills are studied through the use of reaction time. An understanding of the molecular and genetic influences on the speed of responding could be useful in explaining transfer as well as other psychological aspects of human performance. Although the changes in conduction speed due to myelination may be small they could be important because many tasks depend on the precisely timed arrival of information at a neural area and also on the oscillatory synchronization of remote areas (Fields, 2015). These factors could lead to increased response speed as connectivity is changed, since more myelination results in both faster and more reliable conduction speeds. In addition, it may be possible to get a more precise measure of these changes in speed and precision of oscillations due to changes in white matter in humans using MRI to gauge white matter efficiency and EEG to measure the correlation of oscillatory activity or by using the more fine-grained invasive methods made possible with animal models.

Do any of the above ideas illuminate the problem of transfer from the trained task to more general cognitive functions? The issue of transfer is rather specific to network training methods. Since state training does not relate to a specific task its range of influence does not involve transfer, but instead how brain state changes brain activities.

\section{Network Training}

We have argued above that with white matter changes, improvement may be limited to speed and not to other aspects of learning. If correct, this is one restraint on the generality of the effects of training. The speed of activation requirement is not specific to the type of task, but to all tasks using the same network and for which speed or consistency of activation are critical elements of transfer. However, the use of reaction time as an indicant of task performance is very widespread in Cognitive Psychology and many tasks may show enhanced performance from more rapid or reliable conduction between neural areas even when reaction time is not explicitly measured.

Consider training of working memory by the $\mathrm{N}$ back method (Buschkuehl et al., 2014). There is evidence that working memory training strengthens the activation and connections between lateral frontal and parietal areas in the dorsal and ventral attention areas (e.g. frontal eye field, dorsolateral prefrontal cortex connections with the temporal parietal junction and superior parietal lobe). These areas are involved in orienting of attention and more generally in tactical shifts of perspective (Dosenbach et al., 2007) For this reason tasks involving orienting to external stimuli ought to improve even if they use modalities different than the working memory task training as long as the task is improved with faster transmission speed. 
Evidence for far transfer from network training remains inconclusive. Change of white matter pathways would seem to be limited to the domain of the trained task in the case of working memory connections between lateral frontal and parietal areas. However, this pathway is shared by many different cognitive tasks (Duncan \& Owen, 2000), and thus it may be possible to see far transfer across tasks.

\section{State Training}

Although there has been a great deal of interest in different neural states since the development of resting state MRI, there is no clear definition of exactly what methods designed to change brain state such as physical training, meditation or DC stimulation might have in common.

Meditation training involves the cingulo-opercular network and thus one would expect transfer to tasks such as the Stroop and flanker tasks that involve frontal midline rather than lateral brain areas. There have also been many reports of improved learning following DC stimulation over the frontal lobe. The ability of DC stimulation to potentiate the activation of brain networks may lead to a better definition of exactly what is involved in state changes. We believe that our ideas regarding the role of the theta rhythm in improving connectivity following meditation training (Tang et al 2010, 2012) may also relate to DC stimulation. One study found that DC stimulation induced over the frontal cortex produces theta activity that can be recorded subsequently in the resting state (Miller et al., 2015). This study used only a brief period of stimulation, so we do not know whether more sustained stimulation will show a longer lasting effect.

Despite the clear differences between state and network training at the psychological level, the two might involve similar brain mechanisms and also relate to the use of DC stimulation to influence brain activity. All these forms of learning may produce low frequency oscillations during the course of learning and thus enhance white matter. The oscillations may serve to aid in producing long term potentiation and thus improve the synaptic connections between specific cells (Larson, Wong \& Lynch, 1986). Repeated activation of adjacent cells over time could produce an overall improvement in white matter, perhaps first by an increase in axonal density and later in the thickness of the myelin sheath that covers the axons as reported in fMRI studies of meditation (Tang et al., 2012).

The studies reviewed here may point the way toward a deeper understanding of how a given task improves in speed with training. The link between improved connectivity and speed of response suggests one constraint upon how training in a task may change cognition through transfer. The requirement of the transfer task to use the pathways altered by the training may provide a second constraint. We anticipate that continued efforts to connect molecular mechanisms with neural networks of cognition will provide an enlarged context for understanding transfer.

\section{References}

Anderson JR, Fincham JM, Douglass S. Practice and retention: a unifying analysis. Journal of Experimental Psychology: Learning, Memory, \& Cognition. 1999; 25(5):1120-1136. 
Ansari D. Effects of development and enculturation on number representation in the brain. Nature Reviews Neuroscience. 2008; 9(4):278-291. [PubMed: 18334999]

Beirowski B. Concepts for regulation of axon integrity by enwrapping glia. Frontiers in Cellular Neuroscience. 2013; 7:256. [PubMed: 24391540]

Buschkuehl M, Hernandez-Garcia L, Jaeggi SM, Bernard JA, Jonides J. Neural effects of short-term training on working memory. Cognitive, Affective \& Behavioral Neuroscience. 2014; 14(1):147160.

Cohen Kadosh R, Soskic S, Iuculano T, Kanai R, Walsh V. Modulating neuronal activity produces specific and long-lasting changes in numerical competence. Current Biology. 2010; 20(22):20162020. [PubMed: 21055945]

Curran T, Keele S. Attentional and nonattentional forms of sequence learning. Journal of Experimental Psychology. 1993; 19(1):189-202.

Dosenbach NU, Fair DA, Miezin FM, Cohen AL, Wenger KK, Dosenbach RA, et al. Distinct brain networks for adaptive and stable task control in humans. Proceeding of the National Academy of Sciences, USA. 2007; 104(26):11073-11078.

Duncan J, Owen AM. Common regions of the human frontal lobe recruited by diverse cognitive demands. Trends in Neurosciences. 2000; 23(10):475-483. [PubMed: 11006464]

Emery B, Lu QR. Transcriptional and Epigenetic Regulation of Oligodendrocyte Development and Myelination in the Central Nervous System. Cold Spring Harbor Perspectives in Biology. 2015; 7(9):a020461. [PubMed: 26134004]

Fenno L, Yizhar O, Deisseroth K. The development and application of optogenetics. Annual Review of Neuroscience. 2011; 34:389-412.

Fields RD. White matter in learning, cognition and psychiatric disorders. Trends in Neurosciences. 2008; 31(7):361-370. [PubMed: 18538868]

Fields RD. A new mechanism of nervous system plasticity: activity-dependent myelination. Nature Reviews Neuroscience. 2015; 16(12):756-767. [PubMed: 26585800]

Fitts, PM., Posner, MI. Human performance. Belmont, Calif.: Brooks/Cole Pub. Co.; 1967.

Gibson EM, Purger D, Mount CW, Goldstein AK, Lin GL, Wood LS, et al. Neuronal activity promotes oligodendrogenesis and adaptive myelination in the mammalian brain. Science. 2014; 344(6183): 1252304. [PubMed: 24727982]

Harrison TL, Shipstead Z, Hicks KL, Hambrick DZ, Redick TS, Engle RW. Working memory training may increase working memory capacity but not fluid intelligence. Psychological Science. 2013; 24(12):2409-2419. [PubMed: 24091548]

Heathcote A, Brown S, Mewhort DJ. The power law repealed: the case for an exponential law of practice. Psychonomic Bulletin \& Review. 2000; 7(2):185-207. [PubMed: 10909131]

Hillman CH, Erickson KI, Kramer AF. Be smart, exercise your heart: exercise effects on brain and cognition. Nature Reviews Neuroscience. 2008; 9(1):58-65. [PubMed: 18094706]

morphometry. Social, Cognitive and Affective Neuroscience. 3(1):55-61.

Horvath JC, Forte JD, Carter O. Evidence that transcranial direct current stimulation (tDCS) generates little-to-no reliable neurophysiologic effect beyond MEP amplitude modulation in healthy human subjects: A systematic review. Neuropsychologia. 2015; 66:213-236. [PubMed: 25448853]

Jiang J, Zhang Q, van Gaal S. Conflict awareness dissociates theta-band neural dynamics of the medial frontal and lateral frontal cortex during trial-by-trial cognitive control. Neuroimage. 2015; 116:102-111. [PubMed: 25957992]

Larson J, Wong D, Lynch G. Patterned stimulation at the theta frequency is optimal for the induction of hippocampal long-term potentiation. Brain Research. 1986; 368(2):347-350. [PubMed: 3697730]

McKenzie IA, Ohayon D, Li H, de Faria JP, Emery B, Tohyama K, et al. Motor skill learning requires active central myelination. Science. 2014; 346(6207):318-322. [PubMed: 25324381]

Miller J, Berger B, Sauseng P. Anodal transcranial direct current stimulation (tDCS) increases frontalmidline theta activity in the human EEG: a preliminary investigation of non-invasive stimulation. Neuroscience Letters. 2015; 588:114-119. [PubMed: 25576699]

Newell, A., Rosenbloom, PS. Mechanisms of skill acquisition and the law of practice. In: Anderson, JR., editor. Cognitive skills and thier acquisition. Hillsdale, N.J.: Erlbaum; 1981. p. 1-55. 
Olesen PJ, Westerberg H, Klingberg T. Increased prefrontal and parietal activity after training of working memory. Nature Neuroscience. 2004; 7(1):75-79. [PubMed: 14699419]

Osinsky R, Hewig J, Alexander N, Hennig J. COMT Val158Met genotype and the common basis of error and conflict monitoring. Brain Research. 2012; 1452:108-118. [PubMed: 22425188]

Posner, MI., Raichle, ME. Images of Mind. New York: Scientific American Library; 1994.

Posner MI, Tang YY, Lynch G. Mechanisms of white matter change induced by meditation training. Frontiers in Psychology. 2014; 5:1220. [PubMed: 25386155]

Raichle ME. A paradigm shift in functional brain imaging. Journal of Neuroscience. 2009; 29(41): 12729-12734. [PubMed: 19828783]

Reinhart RM, Zhu J, Park S, Woodman GF. Synchronizing theta oscillations with direct-current stimulation strengthens adaptive control in the human brain. Proceeding of the National Academy of Sciences, USA. 2015; 112(30):9448-9453.

Rueda MR, Checa P, Combita LM. Enhanced efficiency of the executive attention network after training in preschool children: immediate changes and effects after two months. Developmental Cognitive Neuroscience. 2012; 2(Suppl 1):S192-204. [PubMed: 22682908]

Schlaggar BL, McCandliss BD. Development of neural systems for reading. Annual Review of Neuroscience. 2007; 30:475-503.

Scholz J, Klein MC, Behrens TE, Johansen-Berg H. Training induces changes in white-matter architecture. Nature Neuroscience. 2009; 12(11):1370-1371. [PubMed: 19820707]

Takeuchi H, Sekiguchi A, Taki Y, Yokoyama S, Yomogida Y, Komuro N, et al. Training of working memory impacts structural connectivity. Journal of Neuroscience. 2010; 30(9):3297-3303. [PubMed: 20203189]

Tang YY, Lu Q, Fan M, Yang Y, Posner MI. Mechanisms of white matter changes induced by meditation. Proceeding of the National Academy of Sciences, USA. 2012; 109(26):10570-10574.

Tang YY, Lu Q, Geng X, Stein EA, Yang Y, Posner MI. Short-term meditation induces white matter changes in the anterior cingulate. Proceeding of the National Academy of Sciences, USA. 2010; 107(35):15649-15652.

Tang YY, Posner MI. Training brain networks and states. Trends in Cognitive Sciences. 2014; 18(7): 345-350. [PubMed: 24816329]

Voelker P, Rothbart MK, Posner MI. A polymorphism related to methylation influences attention during performance of speeded skills. AIMS Neuroscience. 2016; 3(1):40-55.

Voelker P, Sheese BE, Rothbart MK, Posner MI. Methylation polymorphism influences practice effects in children during attention tasks. Journal of Cognitive Neuroscience. 2016

Wang S, Young KM. White matter plasticity in adulthood. Neuroscience. 2014; 276:148-160. [PubMed: 24161723]

Womelsdorf T, Johnston K, Vinck M, Everling S. Theta-activity in anterior cingulate cortex predicts task rules and their adjustments following errors. Proceeding of the National Academy of Sciences, USA. 2010; 107(11):5248-5253.

Xue SW, Tang YY, Tang R, Posner MI. Short-term meditation induces changes in brain resting EEG theta networks. Brain \& Cognition. 2014; 87:1-6. [PubMed: 24632087]

Zatorre RJ, Fields RD, Johansen-Berg H. Plasticity in gray and white: neuroimaging changes in brain structure during learning. Nature Neuroscience. 2012; 15(4):528-536. [PubMed: 22426254] 